\title{
BOUNDEDNESS OF THE MAXIMAL, POTENTIAL AND SINGULAR OPERATORS IN THE GENERALIZED VARIABLE EXPONENT MORREY SPACES
}

\author{
VAGIF S. GULIYEV, JAVANSHIR J. HASANOV and STEFAN G. SAMKO
}

\begin{abstract}
We consider generalized Morrey spaces $\mathscr{M}^{p(\cdot), \omega}(\Omega)$ with variable exponent $p(x)$ and a general function $\omega(x, r)$ defining the Morrey-type norm. In case of bounded sets $\Omega \subset \mathrm{R}^{n}$ we prove the boundedness of the Hardy-Littlewood maximal operator and Calderon-Zygmund singular operators with standard kernel, in such spaces. We also prove a Sobolev-Adams type $\mathscr{M}^{p(\cdot), \omega}(\Omega) \rightarrow \mathscr{M}^{q(\cdot), \omega}(\Omega)$-theorem for the potential operators $I^{\alpha(\cdot)}$, also of variable order. The conditions for the boundedness are given it terms of Zygmund-type integral inequalities on $\omega(x, r)$, which do not assume any assumption on monotonicity of $\omega(x, r)$ in $r$.
\end{abstract}

\section{Introduction}

In the study of local properties of solutions to partial differential equations, together with weighted Lebesgue spaces, Morrey spaces $\mathscr{L}^{p, \lambda}(\Omega)$ play an important role, see [14], [25]. Introduced by C. Morrey [27] in 1938, they are defined by the norm

$$
\|f\|_{\mathscr{L}^{p, \lambda}}:=\sup _{x, r>0} r^{-\frac{\lambda}{p}}\|f\|_{L^{p}(B(x, r))},
$$

where $0 \leq \lambda<n, 1 \leq p<\infty$.

As is known, last two decades there is an increasing interest to the study of variable exponent spaces and operators with variable parameters in such spaces, we refer for instance to the surveying papers [12], [20], [22], [38], on the progress in this field, including topics of Harmonic Analysis and Operator Theory, see also references therein.

Variable exponent Morrey spaces $\mathscr{L}^{p(\cdot), \lambda(\cdot)}(\Omega)$, were introduced and studied in [2] and [29] in the Euclidean setting and in [21] in the setting of metric measure spaces, in case of bounded sets. In [2] there was proved the boundedness of the maximal operator in variable exponent Morrey spaces $\mathscr{L}^{p(\cdot), \lambda(\cdot)}(\Omega)$ under the log-condition on $p(\cdot)$ and $\lambda(\cdot)$ and for potential operators, under the same log-condition and the assumptions $\inf _{x \in \Omega} \alpha(x)>0$,

Received 2 July 2009, in revised form 27 September 2009. 
$\sup _{x \in \Omega}[\lambda(x)+\alpha(x) p(x)]<n$, there was proved a Sobolev type $\mathscr{L}^{p(\cdot), \lambda(\cdot)} \rightarrow$ $\mathscr{L}^{q(\cdot), \lambda(\cdot)}$-theorem. In the case of constant $\alpha$, there was also proved a boundedness theorem in the limiting case $p(x)=\frac{n-\lambda(x)}{\alpha}$, when the potential operator $I^{\alpha}$ acts from $\mathscr{L}^{p(\cdot), \lambda(\cdot)}$ into BMO. In [29] the maximal operator and potential operators were considered in a somewhat more general space, but under more restrictive conditions on $p(x)$. P. Hästö in [18] used his new "local-to-global" approach to extend the result of [2] on the maximal operator to the case of the whole space $\mathrm{R}^{n}$.

In [21] there was proved the boundedness of the maximal operator and the singular integral operator in variable exponent Morrey spaces $\mathscr{L}^{p(\cdot), \lambda(\cdot)}$ in the general setting of metric measure spaces. In the case of constant $p$ and $\lambda$, the results on the boundedness of potential operators and classical CalderonZygmund singular operators go back to [1] and [32], respectively, while the boundedness of the maximal operator in the Euclidean setting was proved in [9]; for further results in the case of constant $p$ and $\lambda$ see for instance [5]- [8].

We introduce the generalized variable exponent Morrey spaces $\mathscr{M}^{p(\cdot), \omega}(\Omega)$ over an open set $\Omega \subseteq \mathrm{R}^{n}$. Generalized Morrey spaces of such a kind in the case of constant $p$ were studied in [4], [13], [26], [28], [30], [31]. Within the frameworks of the spaces $\mathscr{M}^{p(\cdot), \omega}(\Omega)$, over bounded sets $\Omega \subseteq \mathrm{R}^{n}$ we consider the Hardy-Littlewood maximal operator

$$
M f(x)=\sup _{r>0}|B(x, r)|^{-1} \int_{\widetilde{B}(x, r)}|f(y)| d y
$$

potential type operators

$$
I^{\alpha(x)} f(x)=\int_{\Omega}|x-y|^{\alpha(x)-n} f(y) d y, \quad 0<\alpha(x)<n,
$$

the fractional maximal operator

$$
M^{\alpha(x)} f(x)=\sup _{r>0}|B(x, r)|^{\frac{\alpha(x)}{n}-1} \int_{\widetilde{B}(x, r)}|f(y)| d y, \quad 0 \leq \alpha(x)<n
$$

of variable order $\alpha(x)$ and Calderon-Zygmund type singular operator

$$
T f(x)=\int_{\Omega} K(x, y) f(y) d y,
$$

where $K(x, y)$ is a "standard singular kernel", that is, a continuous function defined on $\{(x, y) \in \Omega \times \Omega: x \neq y\}$ and satisfying the estimates

$$
|K(x, y)| \leq C|x-y|^{-n} \quad \text { for all } \quad x \neq y
$$




$$
\begin{array}{ll}
|K(x, y)-K(x, z)| \leq C \frac{|y-z|^{\sigma}}{|x-y|^{n+\sigma}}, & \sigma>0, \text { if }|x-y|>2|y-z|, \\
|K(x, y)-K(\xi, y)| \leq C \frac{|x-\xi|^{\sigma}}{|x-y|^{n+\sigma}}, & \sigma>0, \text { if }|x-y|>2|x-\xi| .
\end{array}
$$

We find the condition on the function $\omega(x, r)$ for the boundedness of the maximal operator $M$ and the singular integral operators $T$ in generalized Morrey space $\mathscr{M}^{p(\cdot), \omega}(\Omega)$ with variable $p(x)$ under the log-condition on $p(\cdot)$. For potential operators, under the same log-condition and the assumptions

$$
\inf _{x \in \Omega} \alpha(x)>0, \quad \sup _{x \in \Omega} \alpha(x) p(x)<n
$$

we also find the condition on $\omega(x, r)$ for the validity of a Sobolev-Adams type $\mathscr{M}^{p(\cdot), \omega}(\Omega) \rightarrow \mathscr{M}^{q(\cdot), \omega}(\Omega)$-theorem, which recovers the known result for the case of the classical Morrey spaces with variable exponents, when $\omega(x, r)=r^{\frac{\lambda(x)-n}{p(x)}}$ and then $\frac{1}{q(x)}=\frac{1}{p(x)}-\frac{\alpha(x)}{n-\lambda(x)}$.

The paper is organized as follows. In Section 2 we provide necessary preliminaries on variable exponent Lebesgue and Morrey spaces. In Section 3 we introduce the generalized Morrey spaces with variable exponents and recall some facts known for generalized Morrey spaces with constant $p$. In Section 4 we deal with the maximal operator, while potential operators are studied in Section 5. In Section 6 we treat Calderon-Zygmund singular operators.

The main results are given in Theorems 4.2, 5.2, 5.5, 6.2. We emphasize that the results we obtain for generalized Morrey spaces are new even in the case when $p(x)$ is constant, because we do not impose any monotonicity type condition on $\omega(x, r)$. The advance in this paper is based on the usage of the approach developed in [15], [16] for constant $p$, and presented for variable $p(x)$ in Theorems 4.1, 5.4, 6.1, and on the estimate of Lemma 2.5.

Notation. $\mathrm{R}^{n}$ is the $n$-dimensional Euclidean space, $\Omega \subseteq \mathrm{R}^{n}$ is an open set, $\ell=\operatorname{diam} \Omega$;

$\chi_{E}(x)$ is the characteristic function of a set $E \subseteq \mathrm{R}^{n}$;

$B(x, r)=\left\{y \in \mathrm{R}^{n}:|x-y|<r\right\}, \widetilde{B}(x, r)=B(x, r) \cap \Omega$;

by $c, C, c_{1}, c_{2}$, etc., we denote various absolute positive constants, which may have different values even in the same line.

\section{Preliminaries on variable exponent Lebesgue and Morrey spaces}

Let $p(\cdot)$ be a measurable function on $\Omega$ with values in $[1, \infty)$. An open set $\Omega$ is assumed to be bounded throughout the whole paper. We suppose that

$$
1<p_{-} \leq p(x) \leq p_{+}<\infty,
$$




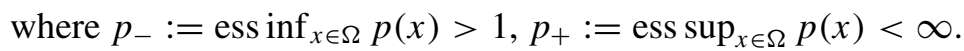

By $L^{p(\cdot)}(\Omega)$ we denote the space of all measurable functions $f(x)$ on $\Omega$ such that

$$
I_{p(\cdot)}(f)=\int_{\Omega}|f(x)|^{p(x)} d x<\infty .
$$

Equipped with the norm

$$
\|f\|_{p(\cdot)}=\inf \left\{\eta>0: I_{p(\cdot)}\left(\frac{f}{\eta}\right) \leq 1\right\},
$$

this is a Banach function space. By $p^{\prime}(\cdot)=\frac{p(x)}{p(x)-1}, x \in \Omega$, we denote the conjugate exponent. The Hölder inequality is valid in the form

$$
\int_{\Omega}\left|f(x)\left\|g(x) \mid d x \leq\left(\frac{1}{p_{-}}+\frac{1}{p_{-}^{\prime}}\right)\right\| f\left\|_{p(\cdot)}\right\| g \|_{p^{\prime}(\cdot)} .\right.
$$

For the basics on variable exponent Lebesgue spaces we refer to [39], [24].

Definition 2.1. By $W L(\Omega)$ (weak Lipschitz) we denote the class of functions defined on $\Omega$ satisfying the log-condition

$$
|p(x)-p(y)| \leq \frac{A}{-\ln |x-y|}, \quad|x-y| \leq \frac{1}{2}, \quad x, y \in \Omega,
$$

where $A=A(p)>0$ does not depend on $x, y$.

THEOREM 2.2 ([10]). Let $\Omega \subset \mathrm{R}^{n}$ be an open bounded set and $p \in W L(\Omega)$ satisfy condition (2.1). Then the maximal operator $M$ is bounded in $L^{p(\cdot)}(\Omega)$.

The following theorem for bounded sets $\Omega$, but for variable $\alpha(x)$, was proved in [37] under the condition that the maximal operator is bounded in $L^{p(\cdot)}(\Omega)$, which became an unconditional result after the result of Diening [10] on maximal operators.

THEOREM 2.3. Let $\Omega \subset \mathrm{R}^{n}$ be bounded, $p, \alpha \in W L(\Omega)$ satisfy assumption (2.1) and the conditions

$$
\inf _{x \in \Omega} \alpha(x)>0, \quad \sup _{x \in \Omega} \alpha(x) p(x)<n .
$$

Then the operator $I^{\alpha(\cdot)}$ is bounded from $L^{p(\cdot)}(\Omega)$ to $L^{q(\cdot)}(\Omega)$ with

$$
\frac{1}{q(x)}=\frac{1}{p(x)}-\frac{\alpha(x)}{n} \text {. }
$$


Singular operators within the framework of the spaces with variable exponents were studied in [11]. From Theorem 4.8 and Remark 4.6 of [11] and the known results on the boundedness of the maximal operator, we have the following statement, which is formulated below for our goals for a bounded $\Omega$, but valid for an arbitrary open set $\Omega$ under the corresponding condition in $p(x)$ at infinity.

THEOREM 2.4 ([11]). Let $\Omega \subset \mathrm{R}^{n}$ be a bounded open set and $p \in W L(\Omega)$ satisfy condition (2.1). Then the singular integral operator $T$ is bounded in $L^{p(\cdot)}(\Omega)$.

We will also make use of the estimate provided by the following lemma (see [36], Corollary to Lemma 3.22).

LEMma 2.5. Let $\Omega$ be a bounded domain and $p$ satisfy the assumption $1 \leq p_{-} \leq p(x) \leq p_{+}<\infty$ and condition (2.2). Let also $\sup v(x)<\infty$ and $\inf [n+v(x) p(x)]>0$. Then

$$
\begin{aligned}
& \left\||x-y|^{\nu(x)} \chi_{B(x, r)}(y)\right\|_{p(y)} \leq C r^{\nu(x)+\frac{n}{p(x)}}, \\
& x \in \Omega, 0<r<\ell=\operatorname{diam} \Omega,
\end{aligned}
$$

where $C$ does not depend on $x$ and $r$.

REMARK 2.6. It may be shown that the constant $C$ in (2.5) may be estimated as $C=C_{0} \ell^{n\left(\frac{1}{p_{-}}-\frac{1}{p_{+}}\right)}$, where $C_{0}$ does not depend on $\Omega$.

Let $\lambda(x)$ be a measurable function on $\Omega$ with values in $[0, n]$. The variable Morrey space $\mathscr{L}^{p(\cdot), \lambda(\cdot)}(\Omega)$ is defined as the set of integrable functions $f$ on $\Omega$ with the finite norm

$$
\|f\|_{\mathscr{L}^{p(\cdot), \lambda(\cdot)}(\Omega)}=\sup _{x \in \Omega, t>0} t^{-\frac{\lambda(x)}{p(x)}}\|f \chi \widetilde{B}(x, t)\|_{L^{p(\cdot)}(\Omega)} .
$$

The following statements are known.

THEOREM 2.7 ([2]). Let $\Omega$ be bounded and $p \in W L(\Omega)$ satisfy condition (2.1) and let a measurable function $\lambda$ satisfy the conditions

$$
0 \leq \lambda(x), \quad \sup _{x \in \Omega} \lambda(x)<n .
$$

Then the maximal operator $M$ is bounded in $\mathscr{L}^{p(\cdot), \lambda(\cdot)}(\Omega)$.

Theorem 2.7 was extended to unbounded domains in [18].

Note that the boundedness of the maximal operator in Morrey spaces with variable $p(x)$ was studied in [21] in the more general setting of quasimetric measure spaces. 
TheOREm 2.8 ([2]). Let $\Omega$ be bounded, $p, \alpha, \lambda \in W L(\Omega)$ and $p$ satisfy condition (2.1). Let also $\lambda(x) \geq 0$ and

$$
\inf _{x \in \Omega} \alpha(x)>0, \quad \sup _{x \in \Omega}[\lambda(x)+\alpha(x) p(x)]<n .
$$

Then the operator $I^{\alpha(\cdot)}$ is bounded from $\mathscr{L}^{p(\cdot), \lambda(\cdot)}(\Omega)$ to $\mathscr{L}^{q(\cdot), \mu(\cdot)}(\Omega)$, where

$$
\frac{1}{q(x)}=\frac{1}{p(x)}-\frac{\alpha(x)}{n} \quad \text { and } \quad \frac{\mu(x)}{q(x)}=\frac{\lambda(x)}{p(x)} .
$$

Theorem 2.9 ([2]). Let $\Omega$ be bounded, $p, \alpha, \lambda \in W L(\Omega)$ and $p$ satisfy condition (2.1). Let also $\lambda(x) \geq 0$ and conditions (2.6) hold. Then the operator $I^{\alpha(\cdot)}$ is bounded from $\mathscr{L}^{p(\cdot), \lambda(\cdot)}(\Omega)$ to $\mathscr{L}^{q(\cdot), \lambda(\cdot)}(\Omega)$, where

$$
\frac{1}{q(x)}=\frac{1}{p(x)}-\frac{\alpha(x)}{n-\lambda(x)}
$$

Theorem 2.10 ([2]). Let $\Omega$ be bounded and $p, \alpha, \lambda \in W L(\Omega)$ satisfy conditions (2.1) and the conditions

$$
\inf _{x \in \Omega} \alpha(x)>0, \quad \lambda(x)+\alpha(x) p(x)=n
$$

hold. Then the operator $M^{\alpha(\cdot)}$ is bounded from $\mathscr{L}^{p(\cdot), \lambda(\cdot)}(\Omega)$ to $L^{\infty}(\Omega)$.

\section{Variable exponent generalized Morrey spaces}

Everywhere in the sequel the functions $\omega(x, r), \omega_{1}(x, r)$ and $\omega_{2}(x, r)$ used in the body of the paper, are non-negative measurable function on $\Omega \times(0, \ell)$, $\ell=\operatorname{diam} \Omega$.

We find it convenient to define the generalized Morrey spaces in the form as follows.

Definition 3.1. Let $1 \leq p<\infty$. The generalized Morrey space $\mathscr{M}^{p(\cdot), \omega}(\Omega)$ is defined by the norm

$$
\|f\|_{M^{p(\cdot), \leq}}=\sup _{x \in \Omega, r>0} \frac{r^{-\frac{n}{p(x)}}}{\omega(x, r)}\|f\|_{L^{p(\cdot)}(\widetilde{B}(x, r))} .
$$

According to this definition, we recover the space $\mathscr{L}^{p(\cdot), \lambda(\cdot)}(\Omega)$ under the choice $\omega(x, r)=r^{\frac{\lambda(x)-n}{p(x)}}$ :

$$
\mathscr{L}^{p(\cdot), \lambda(\cdot)}(\Omega)=\left.\mathscr{M}^{p(\cdot), \omega(\cdot)}(\Omega)\right|_{\omega(x, r)=r^{\frac{\lambda(x)-n}{p(x)}}} .
$$


Everywhere in the sequel we assume that

$$
\inf _{x \in \Omega, r>0} \omega(x, r)>0
$$

which makes the space $\mathscr{M}^{p(\cdot), \omega}(\Omega)$ nontrivial. Note that when $p$ is constant, in the case of $w(x, r) \equiv$ const $>0$, we have the space $L^{\infty}(\Omega)$.

\subsection{Preliminaries on Morrey spaces with constant exponents $p$}

In [15], [16], [28] and [30] there were obtained sufficient conditions on functions $\omega_{1}$ and $\omega_{2}$ for the boundedness of the singular operator $T$ from $\mathscr{M}^{p, \omega_{1}}\left(\mathrm{R}^{n}\right)$ to $\mathscr{M}^{p, \omega_{2}}\left(\mathrm{R}^{n}\right)$. In [30] the following condition was imposed on $w(x, r)$ :

$$
c^{-1} \omega(x, r) \leq \omega(x, t) \leq c \omega(x, r)
$$

whenever $r \leq t \leq 2 r$, where $c(\geq 1)$ does not depend on $t, r$ and $x \in \mathrm{R}^{n}$, jointly with the condition:

$$
\int_{r}^{\infty} \omega(x, t)^{p} \frac{d t}{t} \leq C \omega(x, r)^{p}
$$

for the maximal or singular operator and the condition

$$
\int_{r}^{\infty} t^{\alpha p} \omega(x, t)^{p} \frac{d t}{t} \leq C r^{\alpha p} \omega(x, r)^{p} .
$$

for potential and fractional maximal operators, where $C(>0)$ does not depend on $r$ and $x \in \mathrm{R}^{n}$.

Remark 3.2. Note that the right-hand side inequality in (3.2) may be omitted: it follows from the left-hand-side one and (3.3), which we show in Section 7.

REMARK 3.3. The left-hand side inequality in (3.2) is satisfied for any nonnegative function $w(x, r)$ such that there exists a number $a \in \mathrm{R}^{1}$ such that the function $r^{a} w(x, r)$ is almost increasing in $r$ uniformly in $x$ :

$$
t^{a} w(x, t) \leq c r^{a} w(x, r) \quad \text { for all } \quad 0<t \leq r<\infty
$$

where $c \geq 1$ does not depend on $x, r, t$.

Note that integral conditions of type (3.3) after the paper [3] of 1956 are often referred to as Bary-Stechkin or Zygmund-Bary-Stechkin conditions, see also [17]. The classes of almost monotonic functions satisfying such integral conditions were later studied in a number of papers, see [19], [33], [34] and references therein, where the characterization of integral inequalities of 
such a kind was given in terms of certain lower and upper indices known as Matuszewska-Orlicz indices. Note that in the cited papers the integral inequalities were studied as $r \rightarrow 0$. Such inequalities are also of interest when they allow to impose different conditions as $r \rightarrow 0$ and $r \rightarrow \infty$; such a case was dealt with in [35], [23].

In [30] the following statements were proved.

Theorem 3.4 ([30]). Let $1<p<\infty$ and $\omega(x, r)$ satisfy conditions (3.2)-(3.3). Then the operators $M$ and $T$ are bounded in $\mathcal{M}^{p, \omega}\left(\mathrm{R}^{n}\right)$.

TheOREM 3.5 ([30]). Let $1<p<\infty, 0<\alpha<\frac{n}{p}$, and $\omega(x, t)$ satisfy conditions (3.2) and (3.4). Then the operators $M^{\alpha}$ and $I^{\alpha}$ are bounded from $\mathcal{M}^{p, \omega}\left(\mathrm{R}^{n}\right)$ to $\mathscr{M}^{q, \omega}\left(\mathrm{R}^{n}\right)$ with $\frac{1}{q}=\frac{1}{p}-\frac{\alpha}{n}$.

The following statement, containing the results in [28], [30] was proved in [15] (see also [16]). Note that Theorems 3.6 and 3.7 do not impose condition (3.2).

Theorem 3.6 ([15]). Let $1<p<\infty$ and $\omega_{1}(x, r), \omega_{2}(x, r)$ be positive measurable functions satisfying the condition

$$
\int_{r}^{\infty} \omega_{1}(x, t) \frac{d t}{t} \leq c_{1} \omega_{2}(x, r) .
$$

with $c_{1}>0$ not depending on $x \in \mathrm{R}^{n}$ and $t>0$. Then the operators $M$ and $T$ are bounded from $\mathscr{M}^{p, \omega_{1}(\cdot)}\left(\mathrm{R}^{n}\right)$ to $\mathscr{M}^{p, \omega_{2}(\cdot)}\left(\mathrm{R}^{n}\right)$.

Theorem 3.7 ([15]). Let $0<\alpha<n, 1<p<\infty, \frac{1}{q}=\frac{1}{p}-\frac{\alpha}{n}$ and $\omega_{1}(x, r), \omega_{2}(x, r)$ be positive measurable functions satisfying the condition

$$
\int_{r}^{\infty} t^{\alpha} \omega_{1}(x, t) \frac{d t}{t} \leq c_{1} r^{\alpha} \omega_{2}(x, r)
$$

Then the operators $M^{\alpha}$ and $I^{\alpha}$ are bounded from $\mathscr{M}^{p, \omega_{1}(\cdot)}\left(\mathrm{R}^{n}\right)$ to $\mathscr{M}^{q, \omega_{2}(\cdot)}\left(\mathrm{R}^{n}\right)$.

\section{The maximal operator in the spaces $\mathscr{M}^{p(\cdot), \omega(\cdot)}(\Omega)$}

THEOREM 4.1. Let $\Omega$ be bounded and $p \in W L(\Omega)$ satisfy condition (2.1). Then

$$
\|M f\|_{L^{p())}(\widetilde{B}(x, t))} \leq C t^{\frac{n}{p(x)}} \int_{t}^{\ell} r^{-\frac{n}{p(x)}-1}\|f\|_{L^{p(\cdot)}(\widetilde{B}(x, r))} d r, \quad 0<t<\frac{\ell}{2}
$$

for every $f \in L^{p(\cdot)}(\Omega)$, where $C$ does not depend on $f, x \in \Omega$ and $t$. 
PROof. We represent $f$ as

$$
\begin{aligned}
f=f_{1}+f_{2}, \quad f_{1}(y)=f(y) \chi_{\widetilde{B}(x, 2 t)}(y), & \\
& f_{2}(y)=f(y) \chi_{\Omega \backslash \widetilde{B}(x, 2 t)}(y), \quad t>0,
\end{aligned}
$$

and have

$$
\|M f\|_{L^{p(\cdot)}(\widetilde{B}(x, t))} \leq\left\|M f_{1}\right\|_{L^{p(\cdot)}(\widetilde{B}(x, t))}+\left\|M f_{2}\right\|_{L^{p(\cdot)}(\widetilde{B}(x, t))} .
$$

By Theorem 2.2 we obtain

$$
\left\|M f_{1}\right\|_{L^{p(\cdot)}(\widetilde{B}(x, t))} \leq\left\|M f_{1}\right\|_{L^{p(\cdot)}(\Omega)} \leq C\left\|f_{1}\right\|_{L^{p(\cdot)}(\Omega)}=C\|f\|_{L^{p(\cdot)}(\widetilde{B}(x, 2 t))},
$$

where $C$ does not depend on $f$. From (4.3) we obtain

$$
\begin{aligned}
\left\|M f_{1}\right\|_{L^{p(\cdot)}(\widetilde{B}(x, t))} & \leq C t^{\frac{n}{p(x)}} \int_{2 t}^{\ell} r^{-\frac{n}{p(x)}-1}\|f\|_{L^{p(\cdot)}(\widetilde{B}(x, r))} d r \\
& \leq C t^{\frac{n}{p(x)}} \int_{t}^{\ell} r^{-\frac{n}{p(x)}-1}\|f\|_{L^{p(\cdot)}(\widetilde{B}(x, r))} d r
\end{aligned}
$$

easily obtained from the fact that $\|f\|_{L^{p(\cdot)}(\widetilde{B}(x, 2 t))}$ is non-decreasing in $t$, so that $\|f\|_{L^{p(\cdot)}(\widetilde{B}(x, 2 t))}$ on the right-hand side of (4.3) is dominated by the right-hand side of (4.4). Note that this "complication" of estimate in comparison with (4.3) is done because the term $M f_{2}$ will be estimated below in a similar form, see (4.6).

To estimate $M f_{2}$, we first prove the following auxiliary inequality

$$
\begin{aligned}
& \int_{\Omega \backslash \widetilde{B}(x, t)}|x-y|^{-n} \mid f(y) \mid d y \\
& \leq C \int_{t}^{\ell} s^{-\frac{n}{p(x)}-1}\|f\|_{L^{p(\cdot)}(\widetilde{B}(x, s))} d s, \quad 0<t<\ell .
\end{aligned}
$$

To this end, we choose $\beta>\frac{n}{p_{-}}$and proceed as follows

$$
\begin{aligned}
\int_{\Omega \backslash \widetilde{B}(x, t)}|x-y|^{-n}|f(y)| d y & \\
\quad \leq & \beta \int_{\Omega \backslash \widetilde{B}(x, t)}|x-y|^{-n+\beta}|f(y)|\left(\int_{|x-y|}^{\ell} s^{-\beta-1} d s\right) d y \\
& =\beta \int_{t}^{\ell} s^{-\beta-1}\left(\int_{\{y \in \Omega: 2 t \leq|x-y| \leq s\}}|x-y|^{-n+\beta}|f(y)| d y\right) d s \\
& \leq C \int_{t}^{\ell} s^{-\beta-1}\|f\|_{L^{p(\cdot)}(\widetilde{B}(x, s))}\left\||x-y|^{-n+\beta}\right\|_{L^{p^{\prime}(\cdot)(\widetilde{B}(x, s))}} d s .
\end{aligned}
$$


We then make use of Lemma 2.5 and obtain (4.5).

For $z \in \widetilde{B}(x, t)$ we get

$$
\begin{aligned}
M f_{2}(z) & =\sup _{r>0}|B(z, r)|^{-1} \int_{\widetilde{B}(z, r)}\left|f_{2}(y)\right| d y \\
& \leq C \sup _{r \geq 2 t} \int_{(\Omega \backslash \widetilde{B}(x, 2 t)) \cap \widetilde{B}(z, r)}|y-z|^{-n}|f(y)| d y \\
& \leq C \sup _{r \geq 2 t} \int_{(\Omega \backslash \widetilde{B}(x, 2 t)) \cap \widetilde{B}(z, r)}|x-y|^{-n}|f(y)| d y \\
& \leq C \int_{\Omega \backslash \widetilde{B}(x, 2 t)}|x-y|^{-n}|f(y)| d y .
\end{aligned}
$$

Then by (4.5)

$$
\begin{aligned}
M f_{2}(z) & \leq C \int_{2 t}^{\ell} s^{-\frac{n}{p(x)}-1}\|f\|_{L^{p(\cdot)}(\widetilde{B}(x, s))} d s, \\
& \leq C \int_{t}^{\ell} s^{-\frac{n}{p(x)}-1}\|f\|_{L^{p(\cdot)}(\widetilde{B}(x, s))} d s,
\end{aligned}
$$

where $C$ does not depend on $x, r$. Thus, the function $M f_{2}(z)$, with fixed $x$ and $t$, is dominated by the expression not depending on $z$. Then

$$
\left\|M f_{2}\right\|_{L^{p(\cdot)}(\widetilde{B}(x, t))} \leq C \int_{t}^{\ell} s^{-\frac{n}{p(x)}-1}\|f\|_{L^{p(\cdot)}(\widetilde{B}(x, s))} d s\|1\|_{L^{p(\cdot)}(\widetilde{B}(x, t))} .
$$

Since $\|1\|_{L^{p(\cdot)}(\widetilde{B}(x, t))} \leq C t^{\frac{n}{p(x)}}$ by Lemma 2.5, we then obtain (4.1) from (4.4) and (4.6).

The following theorem extends Theorem 2.7 to the case of generalized Morrey spaces $\mathscr{M}^{p(\cdot), \omega}(\Omega)$.

THEOREM 4.2. Let $\Omega \subset \mathrm{R}^{n}$ be an open bounded set and $p \in W L(\Omega)$ satisfy assumption (2.1) and the function $\omega_{1}(x, r)$ and $\omega_{2}(x, r)$ satisfy the condition

$$
\int_{r}^{\ell} \omega_{1}(x, t) \frac{d t}{t} \leq C \omega_{2}(x, r)
$$

where $C$ does not depend on $x$ and $t$. Then the maximal operator $M$ is bounded from the space $\mathscr{M}^{p(\cdot), \omega_{1}}(\Omega)$ to the space $\mathscr{M}^{p(\cdot), \omega_{2}}(\Omega)$.

Proof. Let $f \in \mathcal{M}^{p(\cdot), \omega_{1}}(\Omega)$. We have

$$
\|M f\|_{M^{p(\cdot), \omega_{2}(\Omega)}}=\sup _{x \in \Omega, t \in(0, \ell)} \omega_{2}^{-1}(x, t) t^{-\frac{n}{p(x)}}\|M f\|_{L^{p(\cdot)}(\widetilde{B}(x, t))} .
$$


The estimation is obvious for $\frac{\ell}{2} \leq t \leq \ell$ in view of (3.1). For

$$
\|M f\|_{\mathscr{M}^{p(\cdot), \omega_{2}(\Omega)}}^{\sim}=\sup _{x \in \Omega, t \in\left(0, \frac{\ell}{2}\right)} \omega_{2}^{-1}(x, t) t^{-\frac{n}{p(x)}}\|M f\|_{L^{p(\cdot)}(\widetilde{B}(x, t))}
$$

by Theorem 4.1 we obtain

$$
\|M f\|_{\mathcal{M}^{p(\cdot), \omega_{2}(\Omega)}}^{\sim} \leq C \sup _{x \in \Omega, 0<t \leq \ell} \omega_{2}^{-1}(x, t) \int_{t}^{\ell} r^{-\frac{n}{p(x)}-1}\|f\|_{L^{p(\cdot)}(\widetilde{B}(x, r))} d r .
$$

Hence

$$
\begin{aligned}
\|M f\|_{\mathscr{M}^{p(\cdot), \omega_{2}}(\Omega)}^{\sim} & \leq C\|f\|_{\mathcal{M}^{p(\cdot), \omega_{1}}(\Omega)} \sup _{x \in \Omega, t \in(0, \ell)} \frac{1}{\omega_{2}(x, t)} \int_{t}^{\ell} \frac{\omega_{1}(x, r)}{r} d r \\
& \leq C\|f\|_{\mathcal{M}^{p(\cdot), \omega_{1}}(\Omega)}
\end{aligned}
$$

by (4.7), which completes the proof.

In the following corollary we recover, from Theorem 4.2, a result obtained in [2] in the case $\omega_{1}(x, r)=\omega_{2}(x, r)=r^{\frac{\lambda(x)-n}{p(x)}}$.

Corollary 4.3. Let $\Omega \subset \mathrm{R}^{n}$ be bounded, $\lambda(x) \geq 0$ and $\sup _{x \in \Omega} \lambda(x)<n$ and $p \in W L(\Omega)$ satisfy condition (2.1). Then the maximal operator $M$ is bounded in the space $\mathscr{L}^{p(\cdot), \lambda(\cdot)}(\Omega)$.

Proof. It suffices to observe that the function $\omega_{1}(x, r)=\omega_{2}(x, r)=r^{\frac{\lambda(x)-n}{p(x)}}$ defining the space $\mathscr{L}^{p(\cdot), \lambda(\cdot)}(\Omega)$, satisfies condition (4.7) under the assumption $\sup _{x \in \Omega} \lambda(x)<n$.

\section{Riesz potential operator in the spaces $\mathcal{M}^{p(\cdot), \omega(\cdot)}(\Omega)$}

In this section we extend Theorem 3.7 to the variable exponent setting. We give two versions of such an extension, one being a generalization of Spanne's result for potential operators, another extending the corresponding Adams' result. Note that Theorems 5.1 and 5.2 in the case of constant exponents $p$ and $\lambda$ were proved in [15] (see also [16]).

\subsection{Spanne type result}

THEOREM 5.1. Let $p, \alpha \in W L(\Omega)$ satisfy condition (2.1) and let $\alpha(x), q(x)$ satisfy the conditions in (2.3) and (2.4). Then

$$
\left\|I^{\alpha(\cdot)} f\right\|_{L^{q(\cdot)}(\widetilde{B}(x, t))} \leq C t^{\frac{n}{q(x)}} \int_{t}^{l} r^{-\frac{n}{q(x)}-1}\|f\|_{L^{p(\cdot)}(\widetilde{B}(x, r))} d r, \quad 0<t \leq \frac{\ell}{2},
$$


where $t$ is an arbitrary number in $\left(0, \frac{\ell}{2}\right)$ and $C$ does not depend on $f, x$ and $t$.

Proof. As in the proof of Theorem 4.1, we represent function $f$ in form (4.2) and have

$$
I^{\alpha(\cdot)} f(x)=I^{\alpha(\cdot)} f_{1}(x)+I^{\alpha(\cdot)} f_{2}(x) .
$$

By Theorem 2.3 we obtain

$$
\left\|I^{\alpha(\cdot)} f_{1}\right\|_{L_{q(\cdot)}(\widetilde{B}(x, t))} \leq\left\|I^{\alpha(\cdot)} f_{1}\right\|_{L_{q(\cdot)}(\Omega)} \leq C\left\|f_{1}\right\|_{L_{p(\cdot)}(\Omega)}=C\|f\|_{L_{p(\cdot)}(\widetilde{B}(x, 2 t))} .
$$

Then

$$
\left\|I^{\alpha(\cdot)} f_{1}\right\|_{L_{q(\cdot)}(\widetilde{B}(x, t))} \leq C\|f\|_{L_{p(\cdot)}(B(\widetilde{x}, 2 t))},
$$

where the constant $C$ is independent of $f$.

Taking into account that

$$
\|f\|_{L_{p(\cdot)}(\widetilde{B}(x, 2 t))} \leq C t^{\frac{n}{q(x)}} \int_{2 t}^{l} r^{-\frac{n}{q(x)}-1}\|f\|_{L_{p(\cdot)}(\widetilde{B}(x, r))} d r,
$$

we get

$$
\left\|I^{\alpha(\cdot)} f_{1}\right\|_{L_{q(\cdot)}(\widetilde{B}(x, t))} \leq C t^{\frac{n}{q(x)}} \int_{2 t}^{l} r^{-\frac{n}{q(x)}-1}\|f\|_{L_{p(\cdot)}(\widetilde{B}(x, r))} d r .
$$

When $|x-z| \leq t,|z-y| \geq 2 t$, we have $\frac{1}{2}|z-y| \leq|x-y| \leq \frac{3}{2}|z-y|$, and therefore

$$
\begin{aligned}
\left\|I^{\alpha(\cdot)} f_{2}\right\|_{L_{q(\cdot)}(\widetilde{B}(x, t))} & \leq\left\|\int_{\Omega \backslash \widetilde{B}(x, 2 t)}|z-y|^{\alpha(y)-n} f(y) d y\right\|_{L_{q(\cdot)}(\widetilde{B}(x, t))} \\
& \leq C \int_{\Omega \backslash \widetilde{B}(x, 2 t)}|x-y|^{\alpha(x)-n}|f(y)| d y\left\|\chi_{\widetilde{B}(x, t)}\right\|_{L_{q(\cdot)}(\Omega)} .
\end{aligned}
$$

We choose $\beta>\frac{n}{q(x)}$ and obtain

$$
\begin{aligned}
& \int_{\Omega \backslash \widetilde{B}(x, 2 t)}|x-y|^{\alpha(x)-n}|f(y)| d y \\
& \quad=\beta \int_{\Omega \backslash \widetilde{B}(x, 2 t)}|x-y|^{\alpha(x)-n+\beta}|f(y)|\left(\int_{|x-y|}^{l} s^{-\beta-1} d s\right) d y \\
& \quad=\beta \int_{2 t}^{l} s^{-\beta-1}\left(\int_{\{y \in \Omega: 2 t \leq|x-y| \leq s\}}|x-y|^{\alpha(x)-n+\beta}|f(y)| d y\right) d s \\
& \leq C \int_{2 t}^{l} s^{-\beta-1}\|f\|_{L_{p(\cdot)}(\widetilde{B}(x, s))}\left\||x-y|^{\alpha(x)-n+\beta}\right\|_{L_{p^{\prime}(\cdot)}(\widetilde{B}(x, s))} d s \\
& \leq C \int_{2 t}^{l} s^{\alpha(x)-\frac{n}{p(x)}-1}\|f\|_{L_{p(\cdot)}(\widetilde{B}(x, s))} d s .
\end{aligned}
$$


Therefore

$$
\left\|I^{\alpha(\cdot)} f_{2}\right\|_{L_{q(\cdot)}(\widetilde{B}(x, t))} \leq C t^{\frac{n}{q(x)}} \int_{2 t}^{l} s^{-\frac{n}{q(x)}-1}\|f\|_{L_{p(\cdot)}(\widetilde{B}(x, s))} d s,
$$

which together with (5.2) yields (5.1).

THEOREM 5.2. Let $\Omega \subset \mathrm{R}^{n}$ be an open bounded set and $p, q \in W L(\Omega)$ satisfy assumption (2.1), $\alpha(x), q(x)$ satisfy the conditions in (2.3), (2.4) and the functions $\omega_{1}(x, r)$ and $\omega_{2}(x, r)$ fulfill the condition

$$
\int_{r}^{\ell} t^{\alpha(x)} \omega_{1}(x, t) \frac{d t}{t} \leq C \omega_{2}(x, r)
$$

where $C$ does not depend on $x$ and $r$. Then the operators $M^{\alpha(\cdot)}$ and $I^{\alpha(\cdot)}$ are bounded from $\mathcal{M}^{p(\cdot), \omega_{1}(\cdot)}(\Omega)$ to $\mathscr{M}^{q(\cdot), \omega_{2}(\cdot)}(\Omega)$.

Proof. Let $f \in \mathscr{M}^{p(\cdot), \omega}(\Omega)$. As usual, when estimating the norm

$$
\left\|I^{\alpha(\cdot)} f\right\|_{M^{q(\cdot), \omega_{2}(\Omega)}}=\sup _{x \in \Omega, t>0} \frac{t^{-\frac{n}{q(x)}}}{\omega_{2}(x, t)}\left\|I^{\alpha(\cdot)} f \chi \widetilde{B}(x, t)\right\|_{L^{q(\cdot)}(\Omega)},
$$

it suffices to consider only the values $t \in\left(0, \frac{\ell}{2}\right)$, thanks to condition (3.1). We estimate $\left\|I^{\alpha(\cdot)} f \chi_{\widetilde{B}(x, t)}\right\|_{L^{q(\cdot)}(\Omega)}$ in (5.4) by means of Theorem 5.1 and obtain

$$
\begin{aligned}
\left\|I^{\alpha(\cdot)} f\right\|_{M^{q(\cdot), \omega_{2}(\Omega)}} & \leq C \sup _{x \in \Omega, t>0} \frac{1}{\omega_{2}(x, t)} \int_{t}^{\ell} r^{-\frac{n}{q(x)}-1}\|f\|_{L^{p(\cdot)}(\widetilde{B}(x, r))} d r \\
& \leq C\|f\|_{M^{p(\cdot), \omega_{1}}(\Omega)} \sup _{x \in \Omega, t>0} \frac{1}{\omega_{2}(x, t)} \int_{t}^{\ell} \frac{r^{\alpha(x)} \omega_{1}(x, r)}{r} d r .
\end{aligned}
$$

It remains to make use of condition (5.3).

In the following corollary we recover the result obtained in [2].

Corollary 5.3. Let $\Omega$ be bounded, $p, \alpha, \lambda \in W L(\Omega)$ and $p$ satisfy condition (2.1). Let also $\lambda(x) \geq 0$ and the conditions (2.3), (2.4), (2.6), (2.7) be fulfilled. Then the operator $\bar{I}^{\alpha(\cdot)}$ is bounded from $\mathscr{L}^{p(\cdot), \lambda(\cdot)}(\Omega)$ to $\mathscr{L}^{q(\cdot), \mu(\cdot)}(\Omega)$.

ProOF. It suffices to observe that the function $\omega_{1}(x, r)=r^{\frac{\lambda(x)-n}{p(x)}}, \omega_{2}(x, r)=$ $r^{\frac{\mu(x)-n}{q(x)}}$ defining the spaces $\mathscr{L}^{p(\cdot), \lambda(\cdot)}(\Omega)$ and $\mathscr{L}^{q(\cdot), \mu(\cdot)}(\Omega)$, satisfies conditions (4.7) and (5.3) under assumption (2.3) and the choice in (2.4), (2.7) for $q(x)$. 


\subsection{Adams type result}

TheOREM 5.4. Let $p \in W L(\Omega)$ satisfy condition (2.1) and let $\alpha(x)$ satisfy the conditions in (2.3). Then

$$
\begin{aligned}
& \left|I^{\alpha(\cdot)} f(x)\right| \leq C t^{\alpha(x)} M f(x) \\
& \quad+C \int_{t}^{l} r^{\alpha(x)-\frac{n}{p(x)}-1}\|f\|_{L^{p(\cdot)}(\widetilde{B}(x, r))} d r, \quad 0<t \leq \frac{\ell}{2},
\end{aligned}
$$

where $t$ is an arbitrary number in $\left(0, \frac{\ell}{2}\right)$ and $C$ does not depend on $f, x$ and $t$.

Proof. As in the proof of Theorem 4.1, we represent the function $f$ in form (4.2) and have

$$
I^{\alpha(\cdot)} f(x)=I^{\alpha(\cdot)} f_{1}(x)+I^{\alpha(\cdot)} f_{2}(x) .
$$

For $I^{\alpha(\cdot)} f_{1}(x)$, following Hedberg's trick (see for instance [37], p. 278, for the case of variable exponents), we obtain $\left|I^{\alpha(\cdot)} f_{1}(x)\right| \leq C_{1} t^{\alpha(x)} M f(x)$. For $I^{\alpha(\cdot)} f_{2}(x)$ we have

$$
\begin{aligned}
\left|I^{\alpha(\cdot)} f_{2}(x)\right| & \leq \int_{\Omega \backslash \widetilde{B}(x, 2 t)}|x-y|^{\alpha(x)-n}|f(y)| d y \\
& \leq C \int_{\Omega \backslash \widetilde{B}(x, 2 t)}|f(y)| d y \int_{|x-y|}^{\infty} r^{\alpha(x)-n-1} d r .
\end{aligned}
$$

Since $\int_{|x-y|}^{\infty} r^{\alpha(x)-n-1} d r \leq C \int_{|x-y|}^{2 \ell} r^{\alpha(x)-n-1} d r$, we obtain

$$
\begin{aligned}
\left|I^{\alpha(\cdot)} f_{2}(x)\right| & \leq C \int_{2 t}^{2 \ell}\left(\int_{2 t<|x-y|<r}|f(y)| d y\right) r^{\alpha(x)-n-1} d r \\
& \leq C \int_{t}^{\ell}\|f\|_{L^{p(\cdot)}(\widetilde{B}(x, r))} r^{\alpha(x)-\frac{n}{p(x)}-1} d r,
\end{aligned}
$$

which proves (5.5).

THEOREM 5.5. Let $p, \alpha \in W L(\Omega)$ satisfy assumption (2.1), $\alpha(x)$ fulfill the conditions in (2.3) and let $\omega(x, t)$ satisfy condition (4.7) and the condition

$$
\int_{r}^{\ell} t^{\alpha(x)-1} \omega(x, t) d t \leq C r^{-\frac{\alpha(x) p(x)}{q(x)-p(x)}}
$$

where $q(x)>p(x)$ and $C$ does not depend on $x \in \Omega$ and $r \in(0, \ell]$. Then the

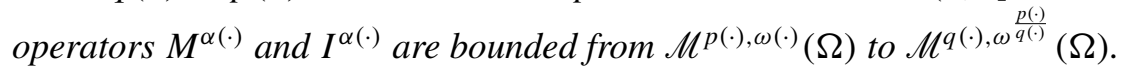

Proof. In view of the well known pointwise estimate $M^{\alpha(\cdot)} f(x) \leq$ $C\left(I^{\alpha(\cdot)}|f|\right)(x)$, it suffices to treat only the case of the operator $I^{\alpha(\cdot)}$. 
Let $f \in \mathcal{M}^{p(\cdot), \omega(\cdot)}(\Omega)$. As in the proof of Theorem 4.2, when estimating the norm

$$
\left\|I^{\alpha(\cdot)} f\right\|_{M^{q(\cdot), \omega}}=\sup _{x \in \Omega, 0<t \leq \ell} \frac{t^{-\frac{n}{q(x)}}}{\omega(x, t)}\left\|I^{\alpha(\cdot)} f \chi_{\widetilde{B}(x, t)}\right\|_{L^{q(\cdot)}(\Omega)},
$$

we may restrict ourselves to the case of $t$ near the origin, $0<t \leq \frac{\ell}{2}$. By Theorem 5.4 we get

$$
\left|I^{\alpha(\cdot)} f(x)\right| \leq C r^{\alpha(x)} M f(x)+C\|f\|_{M^{p(\cdot), \omega}(\Omega)} \int_{r}^{\ell} t^{\alpha(x)-1} \leq(x, t) d t .
$$

Making use of condition (5.6), we obtain

$$
\left|I^{\alpha(\cdot)} f(x)\right| \leq C r^{\alpha(x)} M f(x)+C r^{-\frac{\alpha(x) p(x)}{q(x)-p(x)}}\|f\|_{\mathscr{M}^{p(\cdot), \omega}(\Omega)} .
$$

We then choose $r=\left(\frac{\|f\|_{\mathscr{M} p(\cdot), \omega}}{M f(x)}\right)^{\frac{q(x)-p(x)}{\alpha(x) q(x)}}$ assuming that $f$ is not identical 0 . Hence, for every $x \in \Omega$, we have

$$
\left|I^{\alpha(\cdot)} f(x)\right| \leq C(M f(x))^{\frac{p(x)}{q(x)}}\|f\|_{\mathcal{M}^{p(\cdot), \omega}(\Omega)}^{1-\frac{p(x)}{q(x)}} .
$$

Hence the statement of the theorem follows in view of the boundedness of the maximal operator $M$ in $\mathscr{M}^{p(\cdot), \omega}(\Omega)$ provided by Theorem 4.2 in virtue of condition (4.7).

REMARK 5.6. Let $\omega(x, r) \geq 1$ (which may be supposed by (3.1)). For the exponent $q(x)$, from (5.6) there follows the following bound

$$
\begin{aligned}
& \frac{1}{q(x)} \geq \frac{1}{p(x)}-\frac{\alpha(x)}{m(x)}, \\
& m(x)=p(x)\left[\alpha(x)-\varlimsup_{r \rightarrow 0} \frac{\ln \int_{r}^{\ell} t^{\alpha(x)-1} w(r, t) d t}{\ln r}\right] .
\end{aligned}
$$

The corresponding exponent $q(x)$ given by

$$
\frac{1}{q(x)}=\frac{1}{p(x)}-\frac{\alpha(x)}{m(x)}
$$

might be called the Sobolev-Adams-type exponent corresponding to the space $\mathscr{M}^{p(\cdot), \omega}(\Omega)$. In particular, for the Morrey space $\mathscr{L}^{p(\cdot), \lambda}(\Omega)$ (the case $\omega(x, r)=$ $\left.r^{\frac{\lambda(x)-n}{p(x)}}\right)$, from (5.7) we recover Adams' exponent defined by $\frac{1}{q(x)}=\frac{1}{p(x)}-\frac{\alpha(x)}{n-\lambda(x)}$.

In the following corollary we recover the result obtained in [2]. 
Corollary 5.7. Let $\Omega \subset \mathrm{R}^{n}$ be bounded, $0 \leq \lambda(x)<n, p \in W L(\Omega)$ satisfy condition (2.1), and let (2.6), (2.8) be fulfilled. Then the operators $M^{\alpha(\cdot)}$ and $I^{\alpha(\cdot)}$ are bounded from $\mathscr{L}^{p(\cdot), \lambda(\cdot)}(\Omega)$ to $\mathscr{L}^{q(\cdot), \lambda(\cdot)}(\Omega)$.

Proof. It suffices to observe that the functions $\omega_{1}(x, r)=r^{\frac{\lambda(x)-n}{p(x)}}, \omega_{2}(x, r)$ $=r^{\frac{\lambda(x)-n}{q(x)}}$ defining the space $\mathscr{L}^{p(\cdot), \lambda(\cdot)}(\Omega)$, satisfy conditions $(4.7)$ and (5.6) under assumption (2.6) and the choice of $q(x)$ given in (2.8).

\section{Singular operators in the spaces $\mathcal{M}^{p(\cdot), \omega(\cdot)}(\Omega)$}

Theorems 6.1 and 6.2 proved below, in the case of the constant exponent $p$ were proved in [15] (see also [16]). The boundedness of singular operators in Morrey spaces with variable $p(x)$ was studied in [21] in the case where $w(x, r)=r^{\frac{\lambda(x)-n}{p(x)}}$, but in the more general setting of quasimetric measure spaces.

THEOREM 6.1. Let $\Omega \subset \mathrm{R}^{n}$ be an open bounded set, $p \in W L(\Omega)$ satisfy condition (2.1) and $f \in L^{p(\cdot)}(\Omega)$. Then

(6.1) $\|T f\|_{L^{p(\cdot)}(\widetilde{B}(x, t))} \leq C t^{\frac{n}{p(x)}} \int_{t}^{\ell} r^{-\frac{n}{p(x)}-1}\|f\|_{L^{p(\cdot)}(\widetilde{B}(x, r))} d r, \quad 0<t \leq \frac{\ell}{2}$,

where $C$ does not depend on $f$ and $t$.

Proof. We represent function $f$ as in (4.2) and have

$$
\|T f\|_{L^{p(\cdot)}(\widetilde{B}(x, t))} \leq\left\|T f_{1}\right\|_{L^{p(\cdot)}(\widetilde{B}(x, t))}+\left\|T f_{2}\right\|_{L^{p(\cdot)}(\widetilde{B}(x, t))} .
$$

By Theorem 2.4 we obtain $\left\|T f_{1}\right\|_{L^{p(\cdot)}(\widetilde{B}(x, t))} \leq\left\|T f_{1}\right\|_{L^{p(\cdot)}(\Omega)} \leq C\left\|f_{1}\right\|_{L^{p(\cdot)}(\Omega)}$, so that

$$
\left\|T f_{1}\right\|_{L^{p(\cdot)}(\widetilde{B}(x, t))} \leq C\|f\|_{L^{p(\cdot)}(\widetilde{B}(x, 2 t))} .
$$

Taking into account the inequality

$$
\|f\|_{L^{p(\cdot)}(\widetilde{B}(x, t))} \leq C t^{\frac{n}{p(x)}} \int_{2 t}^{\ell} r^{-\frac{n}{p(x)}-1}\|f\|_{L^{p(\cdot)}(\widetilde{B}(x, r))} d r, \quad 0<t \leq \frac{\ell}{2},
$$

we get

$$
\left\|T f_{1}\right\|_{L^{p(\cdot)}(\widetilde{B}(x, t))} \leq C t^{\frac{n}{p(x)}} \int_{2 t}^{\ell} r^{-\frac{n}{p(x)}-1}\|f\|_{L^{p(\cdot)}(\widetilde{B}(x, r))} d r .
$$

To estimate $\left\|T f_{2}\right\|_{L^{p(\cdot)}(\widetilde{B}(x, t))}$, we observe that

$$
\left|T f_{2}(z)\right| \leq C \int_{\Omega \backslash B(x, 2 t)} \frac{|f(y)| d y}{|y-z|^{n}},
$$


where $z \in B(x, t)$ and the inequalities $|x-z| \leq t,|z-y| \geq 2 t$ imply $\frac{1}{2}|z-y| \leq|x-y| \leq \frac{3}{2}|z-y|$, and therefore

$$
\left\|T f_{2}\right\|_{L^{p(\cdot)}(\widetilde{B}(x, t))} \leq C \int_{\Omega \backslash \widetilde{B}(x, 2 t)}|x-y|^{-n}|f(y)| d y\left\|\chi_{\widetilde{B}(x, t)}\right\|_{\left.L^{p(\cdot)} \Omega\right)} .
$$

Hence by estimate (2.5) (with $v(x) \equiv 0$ ) and inequality (4.5), we get

$$
\left\|T f_{2}\right\|_{L^{p(\cdot)}(\widetilde{B}(x, t))} \leq C t^{\frac{n}{p(x)}} \int_{2 t}^{\ell} r^{-\frac{n}{p(x)}-1}\|f\|_{L^{p(\cdot)}(\widetilde{B}(x, r))} d r .
$$

From (6.2) and (6.3) we arrive at (6.1).

THEOREM 6.2. Let $\Omega \subset \mathrm{R}^{n}$ be an open bounded set, $p \in W L(\Omega)$ satisfy condition (2.1) and $\omega_{1}(x, t)$ and $\omega_{2}(x, r)$ fulfill condition (4.7). Then the singular integral operator $T$ is bounded from the space $\mathscr{M}^{p(\cdot), \omega_{1}}(\Omega)$ to the space $\mathcal{M}^{p(\cdot), \omega_{2}}(\Omega)$.

Proof. Let $f \in \mathcal{M}^{p(\cdot), \omega_{1}}(\Omega)$. As usual, when estimating the norm

$$
\|T f\|_{\mathcal{M}^{p(\cdot), \omega_{2}(\Omega)}}=\sup _{x \in \Omega, t>0} \frac{t^{-\frac{n}{p(x)}}}{\omega_{2}(x, t)}\left\|T f \chi_{\widetilde{B}(x, t)}\right\|_{L^{p(\cdot)}(\Omega)},
$$

it suffices to consider only the values $t \in\left(0, \frac{\ell}{2}\right)$, thanks to condition (3.1). We estimate $\left\|T f \chi_{\widetilde{B}(x, t)}\right\|_{L^{p(\cdot)}(\Omega)}$ in (6.4) by means of Theorem 6.1 and obtain

$$
\begin{aligned}
\|T f\|_{\mathscr{M}^{p(\cdot), \omega_{2}}(\Omega)} & \leq C \sup _{x \in \Omega, t>0} \frac{1}{\omega_{2}(x, t)} \int_{t}^{\ell} r^{-\frac{n}{p(x)}-1}\|f\|_{L^{p(\cdot)}(\widetilde{B}(x, r))} d r \\
& \leq C\|f\|_{\mathscr{M}^{p(\cdot), \omega_{1}}(\Omega)} \sup _{x \in \Omega, t>0} \frac{1}{\omega_{2}(x, t)} \int_{t}^{\ell} \frac{\omega_{1}(x, r)}{r} d r .
\end{aligned}
$$

It remains to make use of condition (4.7).

\section{Appendix}

LEMMA 7.1. If $c^{-1} \omega(x, r) \leq \omega(x, t)$ whenever $0<r \leq t \leq 2 r$, then from (3.3) it follows that the function $\frac{w(x, r)}{r^{n}}$ is almost decreasing uniformly in $x$ :

$$
\frac{w(x, r)}{r^{n}} \geq \frac{1}{2 c C n} \frac{w(x, t)}{t^{n}} \quad \text { for all } \quad 0 \leq r \leq t<\infty,
$$

where $c$ and $C$ are the constant from (3.2) and (3.3) (and consequently the right-hand side inequality in (3.2) holds). 
Proof. From (3.3) we have

$$
\frac{w(x, r)}{r^{n}} \geq \frac{1}{C} \int_{t}^{2 t} \frac{w(x, \tau)}{\tau^{n+1}} d \tau \geq \frac{w(x, t)}{c C} \int_{t}^{2 t} \frac{d \tau}{\tau^{n+1}}
$$

from which (7.1) follows.

AcKnOwLedgements. The research of V. Guliyev and J. Hasanov was partially supported by the grant of BGP II (project ANSF Award / AZM13110-BA-08). The research of S. Samko was supported by Russian Federal Targeted Programme "Scientific and Research-Educational Personnel of Innovative Russia" for 2009-2013, project N 02.740.11.5024.

The authors are thankful to the anonymous referee for the comments which improved the formulations of Theorem 5.5 and Remark 5.6.

\section{REFERENCES}

1. Adams, D. R., A note on Riesz potentials, Duke Math. J. 42 (1975), 765-778.

2. Almeida, A., Hasanov, J. J., Samko, S. G., Maximal and potential operators in variable exponent Morrey spaces, Georgian Math. J. 15 (2008), 195-208.

3. Bary, N. K., and Stechkin, S. B., Best approximations and differential properties of two conjugate functions (Russian), Trudy Moskov. Mat. Obšč. 5 (1956), 483-522.

4. Burenkov, V. I., Guliyev, H. V., Necessary and sufficient conditions for boundedness of the maximal operator in the local Morrey-type spaces, Studia Math. 163 (2004), 157-176.

5. Burenkov, V. I., Guliyev, V. S., Necessary and sufficient conditions for boundedness of the Riesz potential in local Morrey-type spaces, Potential Anal. 31 (2009), 1-39.

6. Burenkov, V. I., Guliyev, H. V., and Guliyev, V. S., Necessary and sufficient conditions for the boundedness of the Riesz potential in the local Morrey-type spaces, Doklady Math. 75 (2007), 103-107; translated from Doklady Akad. Nauk 412 (2007), 585-589.

7. Burenkov, V. I., Guliyev, H. V., and Guliyev, V. S., Necessary and sufficient conditions for boundedness of the fractional maximal operator in local Morrey-type spaces, J. Comput. Appl. Math. 208 (2007), 280-301.

8. Burenkov, V. I., Guliyev, V. S., Serbetci, A., and Tararykova, T. V., Necessary and sufficient conditions for the boundedness of genuine singular integral operators in local Morrey-type spaces (Russian), Doklady Akad. Nauk 422 (2008), 11-14.

9. Chiarenza, F., and Frasca, M., Morrey spaces and Hardy-Littlewood maximal function, Rend. Mat. Appl. (7) 7 (1987), 273-279.

10. Diening, L., Maximal function on generalized Lebesgue spaces $L^{p(x)}$, Math. Inequal. Appl. 7 (2004), 245-253.

11. Diening, L., and Růžička, M., Calderón-Zygmund operators on generalized Lebesgue spaces $L^{p(\cdot)}$ and problems related to fluid dynamics, J. Reine Angew. Math. 563 (2003), 197-220.

12. Diening, L., Hästö, P., and Nekvinda, A., Open problems in variable exponent Lebesgue and Sobolev spaces, pp. 38-58 in: Function Spaces, Differential Operators and Nonlinear Analysis, Proc. Milovy 2004, Math. Inst. Acad. Sci. Czech Republic, Praha 2005.

13. Eridani, A., Gunawan, H., and Nakai, E., On generalized fractional integral operators, Sci. Math. Jpn. 60 (2004), 539-550

14. M. Giaquinta, Multiple integrals in the calculus of variations and nonlinear elliptic systems, Ann. of Math. Studies 105, Princeton Univ. Press, Princeton, NJ 1983. 
15. Guliyev, V. S., Integral operators on function spaces on the homogeneous groups and on domains in $\mathrm{R}^{n}$ (Russian), Doctor's degree dissertation, Mat. Inst. Steklov, Moscow 1994.

16. Guliyev, V. S., Function Spaces, Integral Operators and Two Weighted Inequalities on Homogeneous Groups. Some Applications (Russian), Baku 1999.

17. Guseinov, A. I., and Mukhtarov, Kh. Sh., Introduction to the Theory of Nonlinear Singular Integral Equations (Russian), Nauka, Moscow 1980.

18. P. Hästö, Local-to-global results in variable exponent spaces, Math. Res. Lett. 16 (2009), 263-278.

19. Karapetiants, N. K., and Samko, N. G., Weighted theorems on fractional integrals in the generalized Hölder spaces $H_{0}^{\omega}(\rho)$ via the indices $m_{\omega}$ and $M_{\omega}$, Fract. Calc. Appl. Anal. 7 (2004), 437-458.

20. Kokilashvili, V., On a progress in the theory of integral operators in weighted Banach function spaces, pp. 152-175 in: Function Spaces, Differential Operators and Nonlinear Analysis, Proc. Milovy 2004, Math. Inst. Acad. Sci. Czech Republic, Praha 2005.

21. Kokilashvili, V., and Meskhi, A., Boundedness of maximal and singular operators in Morrey spaces with variable exponent, Arm. J. Math. 1 (2008), 18-28.

22. Kokilashvili, V., and Samko, S., Weighted boundedness of the maximal, singular and potential operators in variable exponent spaces, pp. 139-164 in: A. A. Kilbas and S. V. Rogosin (eds.), Analytic Methods of Analysis and Differential Equations, Proc. Minsk 2006, Cambridge Scientific Publishers, Cambridge 2008.

23. Kokilashvili, V., and Samko, S., Operators of harmonic analysis in weighted spaces with non-standard growth, J. Math. Anal. Appl. 352 (2009), 15-34.

24. Kováčik, O., and Rákosnik, J., On spaces $L^{p(x)}$ and $W^{k, p(x)}$, Czechoslovak Math. J. 41/116 (1991), 592-618.

25. Kufner, A., John, O., and Fučik, S., Function Spaces, Noordhoff, Leyden / Academia, Prague 1977.

26. Kurata, K., Nishigaki, S., and Sugano, S., Boundedness of integral operators on generalized Morrey spaces and Its application to Schrödinger operators, Proc. Amer. Math. Soc. 128 (2000), 1125-1134.

27. Morrey, C. B., On the solutions of quasi-linear elliptic partial differential equations, Trans. Amer. Math. Soc. 43 (1938), 126-166.

28. Mizuhara, T., Boundedness of some classical operators on generalized Morrey spaces, pp. 183-189 in: S. Igari (ed.), Harmonic Analysis, Proc. Sendai 1990, ICM-90 Satellite Conf. Proc., Springer, Tokyo 1991.

29. Mizuta, Y., and Shimomura, T., Sobolev embeddings for Rieszpotentials offunctions in Morrey spaces of variable exponent, J. Math. Soc. Japan 60 (2008), 583-602.

30. Nakai, E., Hardy-Littlewood maximal operator, singular integral operators and Riesz potentials on generalized Morrey spaces, Math. Nachr. 166 (1994), 95-103.

31. Nakai, E., The Campanato, Morrey and Hölder spaces on spaces of homogeneous type, Studia Math. 176 (2006), 1-19.

32. Peetre, J., On the theory of $\mathscr{L}_{p, \lambda}$ spaces, J. Functional Anal. 4 (1969), 71-87.

33. Samko, N., Singular integral operators in weighted spaces with generalized Hölder condition, Proc. A. Razmadze Math. Inst. 120 (1999), 107-134.

34. Samko, N., On non-equilibrated almost monotonic functions of the Zygmund-Bary-Stechkin class, Real Anal. Exchange 30 (2004/2005), 727-745.

35. Samko, N., Samko, S., and Vakulov, B., Weighted Sobolev theorem in Lebesgue spaces with variable exponent, J. Math. Anal. Appl. 335 (2007), 560-583.

36. Samko, S., Convolution type operators in $L^{p(x)}$, Integral Transform. Special Funct. 7 (1998) 123-144.

37. Samko, S., Convolution and potential type operators in the space $L^{p(x)}$, Integral Transform. Special Funct. 7 (1998), 261-284. 
38. Samko, S., On a progress in the theory of Lebesgue spaces with variable exponent: maximal and singular operators, Integral Transform. Special Funct, 16 (2005), 461-482.

39. Sharapudinov, I. I., The topology of the space $\mathscr{L}^{p(t)}([0,1])$, Mat. Zametki $26(1979), 613-$ 632.

\author{
INSTITUTE OF MATHEMATICS AND MECHANICS \\ F.AGAEV STR. 9 \\ AZ 1141 BAKU \\ AZERBAIJAN \\ and \\ AHI EVRAN UNIVERSITY \\ DEPARTMENT OF MATHEMATICS \\ KIRSEHIR \\ TURKEY \\ E-mail: vagif@guliyev.com \\ UNIVERSIDADE DO ALGARVE \\ UCEH \\ CAMPUS DE GAMBELAS \\ PT-8000 FARO \\ PORTUGAL \\ E-mail: ssamko@ualg.pt
}

INSTITUTE OF MATHEMATICS AND MECHANICS F.AGAEV STR. 9

AZ 1141 BAKU

AZERBAIJAN

E-mail: hasanovjavanshir@yahoo.com.tr 